\title{
Isorhapontigenin from Traditionally used Iris \\ Domestica Reduces the Inflammatory Response by Suppressing NF-KB and MAPK Signaling in LPS- Activated RAW 264.7 Macrophages
}

\section{Wen-Chung Huang}

Chang Gung University of Science and Technology

Shu-Ju Wu

Chang Gung University of Science and Technology

Han Lo

Chang Gung University of Science and Technology

Hui-Ling Peng

Chang Gung University of Science and Technology

Sindy Hu

Chang Gung Memorial Hospital Linkou Main Branch: Chang Gung Memorial Hospital

Chian-Jiun Liou ( $\nabla$ ccliu@mail.cgust.edu.tw)

Chang Gung University of Science and Technology https://orcid.org/0000-0002-2949-7060

\section{Research}

Keywords: anti-inflammation, isorhapontigenin, MAPK, NF-KB, proinflammatory cytokines

Posted Date: May 7th, 2021

DOI: https://doi.org/10.21203/rs.3.rs-490290/v1

License: (c) (i) This work is licensed under a Creative Commons Attribution 4.0 International License.

Read Full License 


\section{Abstract}

\section{Background}

Isorhapontigenin, a resveratrol analogue, isolated from Iris domestica can induce apoptosis in tumor cells. Here, we designed to explore whether isorhapontigenin reduced inflammatory response in lipopolysaccharide (LPS)-stimulated RAW 264.7 cells.

\section{Methods}

Isorhapontigenin treated with RAW 264.7 cells, and then with LPS to stimulate inflammatory response. Proinflammatory cytokine expressions were measured using ELISA, and protein expressions were detected using western blots.

Results

Isorhapontigenin significantly inhibited the proinflammatory cytokine expressions. Isorhapontigenin also decreased cyclooxygenase-2 and inducible nitric oxide synthase productions and promoted heme oxygenase-1 expression in LPS-stimulated RAW264.7 cells. Isorhapontigenin could significantly inhibit NF-KB subunit p65 protein localization to the nucleus and reduced MAPK signal pathway activation. Isorhapontigenin also decreased reactive oxygen species production.

\section{Conclusion}

Thus, isorhapontigenin has potential anti-inflammation and anti-oxidative stress that inhibits inflammatory mediators and cytokines expressions through suppressing the MAPK and NF-KB pathways.

\section{Background}

Inflammation can provide a dangerous signal, and inflamed cells or tissues can release inflammatory cytokines or inflammatory mediators to maintain or assist the immune system against microbial invasion [1]. However, excessive release of inflammatory cytokines may lead to dysplasia or continued chronic inflammatory diseases, such as obesity, non-alcoholic steatohepatitis, Alzheimer's disease, atherosclerosis, asthma, and cancer [2]. Therefore, regulating acute and chronic inflammation is of great importance to reducing tissue damage or relieving many chronic diseases. Macrophages are immune cells that are mainly activated by microbial infection or stimulatory molecules [3]. Macrophage activation during microbial infection suppresses the spread of microbes and also stimulates the inflammatory response of macrophages to regulate immune response activation [4]. In gram-negative bacterial infections, lipopolysaccharide (LPS) bind to macrophages, activating toll-like receptor 4 (TLR4) and inducing inflammatory signal pathway expression [5]. Activated macrophages also release excessive inflammatory mediators, chemokines, and cytokines to increase immune cell activation and immune cell function in infected tissues [6]. 
The nuclear transcription factor $\mathrm{KB}(\mathrm{NF}-\mathrm{KB})$ is an important signaling pathway in macrophage-caused inflammatory response [7]. NF-KB is composed of two heterodimeric subunits, p65 and p50. When cells are not receiving a proinflammatory stimulus, p50 and p65 are captured by inhibitor of NF-kB (IKB), which suppresses NF-KB activation. When LPS binds to macrophages, it activates TLR4. This induces IKB phosphorylation, releasing p65 and p50 into the nucleus to switch on the gene expressions of inflammatory cytokines and other inflammatory mediators [8]. In addition, LPS-stimulation of macrophages also induces mitogen-activated protein kinase (MAPK) pathway activation, assisting in the expression of the NF-KB pathway and causing more severe inflammatory responses [9]. Therefore, regulating inflammatory signal pathway expression will improve the inflammatory response of macrophages.

Hemeoxygenase-1 (HO-1) is an antioxidant protein, and can be induced by various stresses that protects cells against oxidative stress [10]. Hence, $\mathrm{HO}-1$ can protect oxidative stress and maintain cellular redox homeostasis [11]. Nuclear factor erythroid 2-related factor 2 (Nrf2) is a transcription factor, and LPS- or inflammatory cytokine-stimulated macrophages will increase Nrf2 translocation into the nucleus to regulate $\mathrm{HO}-1$ expression to protect cells from oxidative damage [12].

Isorhapontigenin is a derivative of resveratrol found in some Chinese herbal medicines [13]. Previous studies pointed out that isorhapontigenin has anti-cancer, anti-oxidant and cardioprotective functions [1416]. Another study found that isorhapontigenin can also inhibit airway epithelial cell inflammatory responses and reduce IL- 6 expression in IL-1 $\beta$-activated A549 cells [17]. Thus, isorhapontigenin is a natural product as an anti-inflammatory agent. However, the molecular mechanism of isorhapontigenin's action on inflammatory macrophages remains unclear. Here, we would evaluate the anti-inflammatory molecular mechanism of isorhapontigenin and investigate inflammatory signaling expressions in RAW264.7 cells stimulated by LPS.

\section{Materials And Methods}

\section{Cell cultures and cell viability}

RAW 264.7 cells cultured in DMEM medium containing $10 \% \mathrm{FBS}$, and incubated at $37^{\circ} \mathrm{C}$ and $5 \% \mathrm{CO} 2$. Isorhapontigenin ( $\geq 98 \%$ purity)(Sigma, St. Louis, MO, USA) dissolved in DMSO solution, and the final DMSO concentration was $\leq 0.1 \%$ in the culture medium. Cell viability detected using CCK-8 assay kit (Sigma). Briefly, RAW 264.7 cells treated with $(0-120 \mu \mathrm{M})$ isorhapontigenin for $24 \mathrm{~h}$. Next, cells added CCK8 solution and cell viability was determined using a microplate spectrophotometer (Multiskan FC, Thermo Scientific, MA, USA).

\section{Proinflammatory cytokine assay}

RAW 264.7 cells treated with $(0-20 \mu \mathrm{M})$ isorhapontigenin for $1 \mathrm{~h}$. Next, $100 \mathrm{ng} / \mathrm{mL}$ LPS (Escherichia coli 055:B5, Sigma) stimulated the cells, and cultured for $8 \mathrm{~h}$ to detect IL-6 and TNF-a expression, and cultured $24 \mathrm{~h}$ to detect $\mathrm{PGE}_{2}$ and IL-1 $\beta$ production by ELISA kits (R\&D Systems, Minneapolis, MN, USA). 
SP600125 (JNK inhibitor), SB203580 (p38 inhibitor), and PD98059 (ERK inhibitor) (Sigma) treated with or without $10 \mu \mathrm{M}$ isorhapontigenin to assay IL-6 and TNF-a production. Specific cytokine or PGE 2 were determined using a microplate spectrophotometer (Thermo Scientific).

\section{Nitrite assay}

Cells treated with isorhapontigenin $(0-20 \mu \mathrm{M})$ for $1 \mathrm{~h}$. Next, cells stimulated with $100 \mathrm{ng} / \mathrm{mL}$ LPS (Sigma) for $24 \mathrm{~h}$. The supernatant treated with the Griess reagent (Sigma) for $15 \mathrm{~min}$, and nitrite levels was detected at $570 \mathrm{~nm}$, a microplate spectrophotometer (Thermo Scientific).

\section{Preparation of total and nuclear protein}

Cells treated with isorhapontigenin for $1 \mathrm{~h}$. Next, cells stimulated with or without $100 \mathrm{ng} / \mathrm{mL}$ LPS for 30 min to detect protein phosphorylation, or for $24 \mathrm{~h}$ to investigate total protein expression. Briefly, cells collected and lysed using protein lysis buffer containing phosphatase inhibitor cocktail (Thermo Scientific). Furthermore, nuclear proteins isolated using NE-PER cell fractionation kits (Thermo Scientific).

\section{Western blotting}

Protein separated using SDS-PAGE gel and the protein bands transferred to PVDF membranes. The membranes incubated with specific antibodies, including ERK1/2, JNK, p38, ICAM-1, inducible nitric oxide synthase (iNOS), phosphorylated-ERK 1/2, phosphorylated-JNK, and phosphorylated p38 (Cell Signaling, MA, USA), cyclooxygenase-2 (COX-2), and $\beta$-actin (Sigma), IKB-a, lamin B1, HO-1, phosphorylated-IKB-a, and Nrf2 (Santa Cruz, CA, USA). Following washing, membranes incubated with secondary antibodies, and finally proteins were presented using enhanced chemiluminescence solution (Thermo Scientific) and the BioSpectrum imaging system (UVP, Upland, CA, USA).

\section{ROS expression}

Cells treated with isorhapontigenin for $1 \mathrm{~h}$, then cells stimulated with LPS for $24 \mathrm{~h}$. Cells washed and added with DCFH-DA as described previously [18]. ROS expressions observed using fluorescence microscopy (Olympus). Cells also detected ROS levels using the multimode plate readers (BioTek synergy $\mathrm{HT}$ ). Furthermore, MAPK inhibitors (Enzo Life Sciences) were treated with or without $10 \mu \mathrm{M}$ isorhapontigenin to detect ROS expression.

\section{Immunofluorescence staining}

Cells were used to seed microscope slides in culture plates and incubated with isorhapontigenin for $1 \mathrm{~h}$, then LPS stimulated cells for $24 \mathrm{~h}$. The slides treated with a specific P65 antibody (1:100), followed by a fluorescent secondary antibody. Finally, the slides treated with DAPI solution for nuclear staining and presented images using fluorescence microscope (Olympus).

\section{Statistics}


Experimental results performed at least three independent experiments. Data are presented as the mean \pm SD. Statistical analysis of experimental data performed using one-way ANOVA followed by Dunnett's test and post hoc analysis. Statistical significance defined as $p<0.05$.

\section{Results}

\section{Effects of isorhapontigenin on cell viability}

Isorhapontigenin did not effectively affect cell viability at concentrations $\leq 40 \mu \mathrm{M}$ (Fig. 1a). Hence, cellular experiments used concentrations of isorhapontigenin from $0-20 \mu \mathrm{M}$.

\section{Isorhapontigenin decreased proinflammatory cytokines expressions}

10-20 $\mu \mathrm{M}$ isorhapontigenin had an inhibitory effect on IL-1 $\beta$ concentrations in LPS-stimulated RAW 264.7 cells (Fig. 1b). Furthermore, isorhapontigenin also significantly reduced the IL-6 and TNF-a levels in a concentration-dependent manner (Fig. 1c-d).

\section{Isorhapontigenin decreased COX-2 and iNOSexpression}

Isorhapontigenin significantly suppressed nitrite and $\mathrm{PGE}_{2}$ expressions (Fig. 2a-b). Isorhapontigenin also significantly attenuated COX-2 and iNOS expression compared to LPS-induced RAW 264.7 cells (Fig. 2ce).

\section{Isorhapontigenin attentuates IKBa phosphorylation and NF-KB activation}

The results demonstrated that isorhapontigenin significantly reduced IкB-a degradation and phosphorylation compared to LPS-incubated RAW 264.7 cells (Fig. 3). Furthermore, p65 was mostly cytoplasmic in LPS unstimulated cells, while its level in nucleus increased after treatment with LPS. Interestingly, western blotting of cytosolic and nuclear fractions demonstrated that the nuclear translocation of p65 decreased in isorhapontigenin-treated compared with untreated LPS-induced cells (Fig. $4 a-c)$, and comparison of fluorescent staining of p65 in cells under these conditions confirmed the significant suppression of nuclear translocation of p65 by isorhapontigenin (Fig. 4d).

\section{Isorhapontigenin regulated the phosphorylation of MAPK pathway}

Isorhapontigenin effectively decreased the phosphorylation of ERK1/2, JNK, and p38 (Fig. 5a-b).

Therefore, we used MAPK inhibitors to investigate how isorhapontigenin attenuates proinflammatory cytokine expression. The resulted demonstrated that isorhapontigenin enhanced the ability of MAPK inhibitors to suppress IL-6 and TNF-a expression, respectively (Fig. 5c-d).

\section{Isorhapontigenin increased Nrf2 and HO-1 production}

We found that isorhapontigenin significantly promoted HO-1 levels in cytoplasm and increased Nrf2 expressions in nucleus compared with LPS-stimulated macrophages (Fig. 6a-c). In cells treated with 
DCFH-DA, fluorescence microscopy revealed that isorhapontigenin could decrease intracellular ROS expressions (Fig. 6d). RAW 264.7 cells also incubated with DCFH-DA solution, and detected ROS expressions using Multi-Mode microplate reader. The result demonstrated that isorhapontigenin effectively reduced ROS expressions in LPS-stimulated RAW 264.7 cells (Fig. 6e). Furthermore, when isorhapontigenin added with MAPK inhibitors, ROS productions effectively decreased more effectively compared to isorhapontigenin treated with LPS-activated RAW 264.7 cells (Fig. 6f).

\section{Discussion}

Isorhapontigenin is a tetrahydroxylated stilbenoid isolated from I. domestica, G. cleistostachyum, and wine grapes [19]. Isorhapontigenin has been known to have anticancer, anti-inflammatory, antioxidant effects [14,20,21]. Isorhapontigenin was found that could inhibited cartilage matrix damage and chondrocyte inflammation in rats, suppressing COX-2 and iNOS expression in IL-1 $\beta$-induced chondrocytes [17]. However, anti-inflammatory molecular mechanism of isorhapontigenin on immune cells is not yet fully clear. In this current study, isorhapontigenin significantly reduced pro-inflammatory cytokine productions by LPS-stimulated macrophages. In addition, in LPS-stimulated macrophages, isorhapontigenin also reduced iNOS and COX-2 productions, and increased HO-1 and Nrf2 protein expression. We also found that isorhapontigenin significantly attenuated the nuclear translocation of p65 and decreased MAPK phosphorylation in inflammatory signaling pathways, and improved the nuclear translocation of Nrf2 to promote its antioxidant effect in LPS-induced macrophages.

When infecting bacteria invade human tissues or organs, innate immune system detect and devour them, preventing bacterial spread, and reducing the likelihood of diseases such as sepsis and bacteremia [5]. The LPS binds to the TLR4 receptor of macrophages, activated inflammatory signaling and released inflammatory mediators and cytokines to promote the ability of immune cells to resist bacterial infection [22]. LPS-stimulated macrophages can induce excessive iNOS expression, and iNOS can convert Larginine to L-citrulline and produce NO [23]. These NO molecules in turn activate inflammatory molecular messages to inform inflammatory cells and tissues of danger, interfering with the normal physiological functions of tissues [24]. Previous research found that isorhapontigenin could reduce NO and iNOS expression in IL-1 $\beta$-induced rat chondrocytes [17]. In this study, isorhapontigenin significantly inhibited iNOS expression in LPS-stimulated macrophages, and thus could reduce the expression of inflammatory signaling pathways caused by excessive NO. In addition, LPS-stimulated macrophages can also strongly increase COX-2 expression [25]. COX-2 metabolizes arachidonic acid to produce $\mathrm{PGE}_{2}$, which increases pain in inflamed tissue and causes vasodilation, allowing increased immune-cell migration into the tissue [26]. COX-2 inhibitors as anti-inflammatory and analgesic drugs can decrease $\mathrm{PGE}_{2}$ production by inhibiting COX-2 expression, thus reducing inflammation [26]. Isorhapontigenin was reported to reduce COX-2 and $P G E_{2}$ expression and reduce IL-1 $\beta$-induced rat cartilage explant damage [17]. Our experiments also found that isorhapontigenin inhibits COX-2 and PGE 2 expressions in LPS-activated macrophages. Hence, isorhapontigenin has the ability to reduce secretions of macrophage inflammatory mediator and thus reduce the excessive activation of immune cells. 
During bacterial infection, activated macrophages release inflammatory cytokines, which cause more immune cell activation and severe inflammation [27]. LPS-stimulated macrophages can express high levels of TNF-a to increase the permeability of vascular endothelial cells and promote their expression of cell adhesion molecules. This allows more neutrophils or monocytes to infiltrate the infected tissue to promote immunity and attack microorganisms [28]. However, bacterial infection induces excessive activation of macrophages, which release excessive amounts of TNF-a, causing fever, lowered blood pressure, and even organ necrosis, sepsis, and death [22, 29]. Therefore, reducing the uncontrolled activation of macrophages would reduce the excessive production of TNF-a and reduce the risk of death from organ failure and shock [30]. In this study, 5-20 $\mu \mathrm{M}$ isorhapontigenin inhibited TNF-a production in LPS-induced macrophages. Isorhapontigenin is an analog of resveratrol. Previously, scholars found that 1-25 $\mu \mathrm{M}$ resveratrol can reduce the secretion of TNF-a by LPS-stimulated macrophages [31]. Therefore, we thought that isorhapontigenin should also have a good anti-inflammatory effect. Inflamed macrophages also release a large amount of IL-6. IL-6 is considered to be an important factor in the expression of C-reactive protein, which initiates acute inflammation [32]. In some patients with chronic obstructive pulmonary disease, the lungs contain large amounts of C-reactive protein and IL-6, which stimulate vasodilation and aggravate inflammation [33]. In addition, LPS-induced macrophages secrete $\mathrm{IL}-1 \beta$ to stimulate the hypothalamus to increase body temperature and promote the innate immune response against bacterial infection [34]. IL-1 $\beta$ can also induce lung neutrophil infiltration and increase lung inflammation in mice with LPS-induced lung injury [35]. This study also found that 10-20 $\mu \mathrm{M}$ isorhapontigenin inhibited the production of IL- $1 \beta$ and IL- 6 by inflammatory macrophages. Therefore, isorhapontigenin may be a natural anti-inflammatory compound that can reduce inflammation during bacterial infections.

$\mathrm{NF}-\mathrm{kB}$ can be translocated to the nucleus where it induces the expression of inflammation-related genes, including those encoding proinflammatory cytokines, iNOS, and COX-2 [7]. LPS binds to the CD14 molecule on macrophages, activating TLR4, which triggers signaling through the NF-KB pathway [36, 37]. In inactivated macrophages, IкB (composed of IкB- $\alpha$ and IкB- $\beta$ subunits) inhibits NF-kB activity, restricting it to the cytoplasm. LPS stimulates IкB phosphorylation and release, allowing NF-kB to enter the nucleus to drive the expression of inflammation-related gene expression [36]. In this current study, isorhapontigenin inhibited IKB phosphorylation and suppressed IKB degradation. Therefore, in inflammatory macrophages treated with isorhapontigenin, most NF-kB remained in the cytoplasm, attenuating the expression of inflammation-related genes. To further confirm that isorhapontigenin can reduce NF-kB translocation into the nucleus of inflamed macrophages, we used immunofluorescent staining to demonstrate that isorhapontigenin treatment reduces p65 protein expression in the nucleus of LPS-stimulated macrophages. Recent studies demonstrated that the NF-KB pathway is important in regulating the increased expression of COX-2 and iNOS in activated macrophages [38]. Previous studies have found that MAPK signaling also promotes NF-KB activity in these calls. We found that isorhapontigenin treatment of LPS-stimulated macrophages significantly reduced JNK, ERK 1/2, and p38 phosphorylation. Additionally, MAPK inhibitors co-cultured with isorhapontigenin and found that isorhapontigenin addition decreased proinflammatory cytokine expression. Thus, our results show that 
isorhapontigenin reduces the expression of inflammatory mediators and cytokines by inhibiting the NF$\mathrm{KB}$ and MAPK pathways.

HO-1 protein expression is induced under various stresses. Stimulation of macrophages by inflammation or oxidative molecules induces massive expression of HO-1 [39]. HO-1 metabolizes heme to produce carbon monoxide, biliverdin, and iron [10]. Resveratrol can stimulate macrophages to express a large amount of HO-1, inhibit the production of iNOS, COX-2, and TNF-a, and reduce the expression of ROS in LPS-induced macrophages [31]. Recent studies demonstrated that Nrf2 is an essential transcription factor for regulated antioxidant pathway [40]. Nrf2 translocation into the nucleus to regulate the expression of HO-1, which enhances the cell's antioxidant ability [12]. In addition, isorhapontigenin added together with MAPK inhibitors could significantly attenuate ROS productions in LPS-stimulated macrophages. Therefore, our results indicate that isorhapontigenin promotes the nuclear translocation of Nrf2 and induces HO-1 synthesis in macrophages, thus reducing the levels of ROS via blocked MAPK signal pathways in LPS-stimulated macrophages.

\section{Conclusion}

In conclusion, isorhapontigenin reduced proinflammatory cytokine and mediator expression by suppressing of the activation of the MAPK and NF-KB signaling pathways in LPS-stimulated macrophages. Isorhapontigenin could decreased ROS levels by upregulating the Nrf2/HO-1 pathway (Figure 7). Hence, isorhapontigenin has anti-inflammatory and antioxidant activity and is a potential novel anti-inflammatory agent.

\section{Declarations}

\section{COMPLIANCE WITH ETHICAL STANDARDS}

\section{Ethics Approval and Consent to Participate}

Not applicable

\section{Consent for publication}

Not applicable

\section{Availability of data and materials}

The data that support the findings of this study are available from the corresponding author upon reasonable request.

\section{Competing interests}

The authors have no conflicts of interest to declare. 


\section{Funding}

This study was supported in part by grants from the Chang Gung Memorial Hospital (CMRPF1F0083, CMRPF1G0181, CMRPF1H0023, and CMRPF1H0043) and the Chang Gung University of Science and Technology (ZRRPF3K0011 and ZRRPF3K0111-7), and the Ministry of Science and Technology in Taiwan (109-2320-B-255-006-MY3).

\section{Authors' contributions}

Designed and performed the experiments: $\mathrm{WCH}, \mathrm{SH}$, and CJL; Analysis and interpretation of data: WCH, SJW, HL and HLP; Drafting the manuscript: SH, and CJL.

\section{Acknowledgements}

Not applicable

\section{References}

1. Tursi A, Elisei W. Role of inflammation in the pathogenesis of diverticular disease. Mediators Inflamm. 2019, 2019:8328490.

2. Suzuki K. Chronic inflammation as an immunological abnormality and effectiveness of exercise. Biomolecules. 2019, 9(6):223.

3. Hine AM, Loke P. Intestinal Macrophages in Resolving Inflammation. J Immunol. 2019, 203(3):593599.

4. Griffin TM, Scanzello CR. Innate inflammation and synovial macrophages in osteoarthritis pathophysiology. Clin Exp Rheumatol. 2019, 37 Suppl 120(5):57-63.

5. Rossol M, Heine H, Meusch U, Quandt D, Klein C, Sweet MJ, Hauschildt S. LPS-induced cytokine production in human monocytes and macrophages. Critical reviews in immunology. 2011, 31(5):379446.

6. Sharif O, Brunner JS, Vogel A, Schabbauer G. Macrophage rewiring by nutrient associated PI3K dependent pathways. Front Immunol. 2019, 10:2002.

7. Mussbacher M, Salzmann M, Brostjan C, Hoesel B, Schoergenhofer C, Datler H, Hohensinner P, Basílio $J$, Petzelbauer P, Assinger A et al. Cell type-specific roles of NF-kB linking inflammation and thrombosis. Front Immunol. 2019, 10:85.

8. Mitchell JP, Carmody RJ. NF-KB and the Transcriptional Control of Inflammation. Int Rev Cell Mol Biol. 2018, 335:41-84.

9. Yeung YT, Aziz F, Guerrero-Castilla A, Arguelles S. Signaling pathways in inflammation and antiinflammatory therapies. Curr Pharm Des. 2018, 24(14):1449-1484.

10. Facchinetti MM. Heme-Oxygenase-1. Antioxid Redox Signal. 2020, 32(17):1239-1242. 
11. Zhu X, Fan WG, Li DP, Kung H, Lin MC. Heme oxygenase-1 system and gastrointestinal inflammation: a short review. World journal of gastroenterology : WJG. 2011, 17:4283-4288.

12. Habtemariam S. The Nrf2/HO-1 Axis as Targets for Flavanones: Neuroprotection by Pinocembrin, Naringenin, and Eriodictyol. Oxid Med Cell Longev. 2019, 2019:4724920.

13. Ravishankar D, Albadawi DAl, Chaggar V, Patra PH, Williams HF, Salamah M, Vaiyapuri R, Dash PR, Patel K, Watson KA et al. Isorhapontigenin, a resveratrol analogue selectively inhibits ADP-stimulated platelet activation. European journal of pharmacology. 2019, 862:172627.

14. Abbas AM. Cardioprotective effect of resveratrol analogue isorhapontigenin versus omega-3 fatty acids in isoproterenol-induced myocardial infarction in rats. J Physiol Biochem. 2016, 72(3):469-484.

15. Jiang G, Wu AD, Huang C, Gu J, Zhang L, Huang H, Liao X, Li J, Zhang D, Zeng X et al. Isorhapontigenin (ISO) Inhibits Invasive bladder cancer formation in vivo and human bladder cancer invasion in vitro by targeting STAT1/FOXO1 axis. Cancer Prev Res (Phila). 2016, 9(7):567-580.

16. Lu Y, Wang A, Shi P, Zhang H. A theoretical study on the antioxidant activity of piceatannol and isorhapontigenin scavenging nitric oxide and nitrogen dioxide radicals. PLoS One. 2017, 12(1): $\mathrm{e} 0169773$.

17. Ma Y, Tu C, Liu W, Xiao Y, Wu H. Isorhapontigenin Suppresses Interleukin-1 $\beta$-Induced Inflammation and Cartilage Matrix Damage in Rat Chondrocytes. Inflammation. 2019, 42(6):2278-2285.

18. Liou CJ, Chen YL, Yu MC, Yeh KW, Shen SC, Huang WC. Sesamol alleviates airway hyperresponsiveness and oxidative stress in asthmatic mice. Antioxidants (Basel). 2020, 9(4):295.

19. Li HL, Wang AB, Huang Y, Liu DP, Wei C, Williams GM, Zhang CN, Liu G, Liu YQ, Hao DL et al. Isorhapontigenin, a new resveratrol analog, attenuates cardiac hypertrophy via blocking signaling transduction pathways. Free Radic Biol Med. 2005, 38(2):243-257.

20. Fernández-Marín MI, Guerrero RF, García-Parrilla MC, Puertas B, Richard T, Rodriguez-Werner MA, Winterhalter P, Monti JP, Cantos-Villar E. Isorhapontigenin: a novel bioactive stilbene from wine grapes. Food Chem. 2012, 135(3):1353-1359.

21. Subedi L, Teli MK, Lee JH, Gaire BP, Kim MH, Kim SY. A stilbenoid isorhapontigenin as a potential anti-cancer agent against breast cancer through inhibiting sphingosine kinases/tubulin stabilization. Cancers (Basel). 2019, 11(12):1947.

22. Rathinam VAK, Zhao Y, Shao F. Innate immunity to intracellular LPS. Nat Immunol. 2019, 20(5):527533.

23. Rosadini CV, Kagan JC. Early innate immune responses to bacterial LPS. Curr Opin Immunol. 2017, 44:14-19.

24. Płóciennikowska A, Hromada-Judycka A, Borzęcka K, Kwiatkowska K. Co-operation of TLR4 and raft proteins in LPS-induced pro-inflammatory signaling. Cell Mol Life Sci. 2015, 72(3):557-581.

25. Cochet F, Peri F. The Role of Carbohydrates in the Lipopolysaccharide (LPS)/Toll-Like Receptor 4 (TLR4) Signalling. Int J Mol Sci. 2017, 18(11). 
26. Rawat C, Kukal S, Dahiya UR, Kukreti R. Cyclooxygenase-2 (COX-2) inhibitors: future therapeutic strategies for epilepsy management. J Neuroinflammation. 2019, 16(1):197.

27. Hooftman A, O'Neill LAJ. The Immunomodulatory Potential of the Metabolite Itaconate. Trends Immunol. 2019, 40(8):687-698.

28. Pan W, Wang Q, Chen Q. The cytokine network involved in the host immune response to periodontitis. Int J Oral Sci. 2019, 11(3):30.

29. Song B, Zhang YL, Chen LJ, Zhou T, Huang WK, Zhou X, Shao LQ. The role of Toll-like receptors in periodontitis. Oral Dis. 2017, 23(2):168-180.

30. Chong DL, Sriskandan S. Pro-inflammatory mechanisms in sepsis. Contrib Microbiol. 2011, 17:86107.

31. Zong Y, Sun L, Liu B, Deng Y, Zhan D, Chen Y, He Y, Liu J, Zhang Z, Sun J et al. Resveratrol inhibits LPS-induced MAPKs activation via activation of the phosphatidylinositol 3-kinase pathway in murine RAW 264.7 macrophage cells. PLoS One. 2012, 7:e44107.

32. Tanaka T, Narazaki M, Masuda K, Kishimoto T. Regulation of IL-6 in immunity and diseases. Adv Exp Med Biol. 2016, 941:79-88.

33. Nakamoto $K$, Watanabe $M$, Sada $M$, Inui T, Nakamura $M$, Honda $K$, Wada H, Ishii $H$, Takizawa $H$. Pseudomonas aeruginosa-derived flagellin stimulates IL-6 and IL-8 production in human bronchial epithelial cells: A potential mechanism for progression and exacerbation of COPD. Exp Lung Res. 2019, 45(8):255-266.

34. Yazdi AS, Ghoreschi K. The Interleukin-1 Family. Adv Exp Med Biol. 2016, 941:21-29.

35. Jia X, Cao B, An Y, Zhang X, Wang C. Rapamycin ameliorates lipopolysaccharide-induced acute lung injury by inhibiting IL-1 $\beta$ and IL-18 production. Int Immunopharmacol. 2019, 67:211-219.

36. Mulero MC, Huxford T, Ghosh G. NF-KB, IKB, and IKK: Integral components of immune system signaling. Adv Exp Med Biol. 2019, 1172:207-226.

37. Durand JK, Baldwin AS. Targeting IKK and NF-KB for Therapy. Adv Protein Chem Struct Biol. 2017, 107:77-115.

38. Morrison DK. MAP kinase pathways. Cold Spring Harb Perspect Biol. 2012, 4(11):a011254.

39. Chen S, Wang X, Nisar MF, Lin M, Zhong JL. Heme Oxygenases: Cellular Multifunctional and Protective Molecules against UV-Induced Oxidative Stress. Oxid Med Cell Longev. 2019, 2019:5416728.

40. Zhang J, Wang X, Vikash V, Ye Q, Wu D, Liu Y, Dong W. ROS and ROS-Mediated Cellular Signaling. Oxid Med Cell Longev. 2016, 2016:4350965.

\section{Figures}


(a)

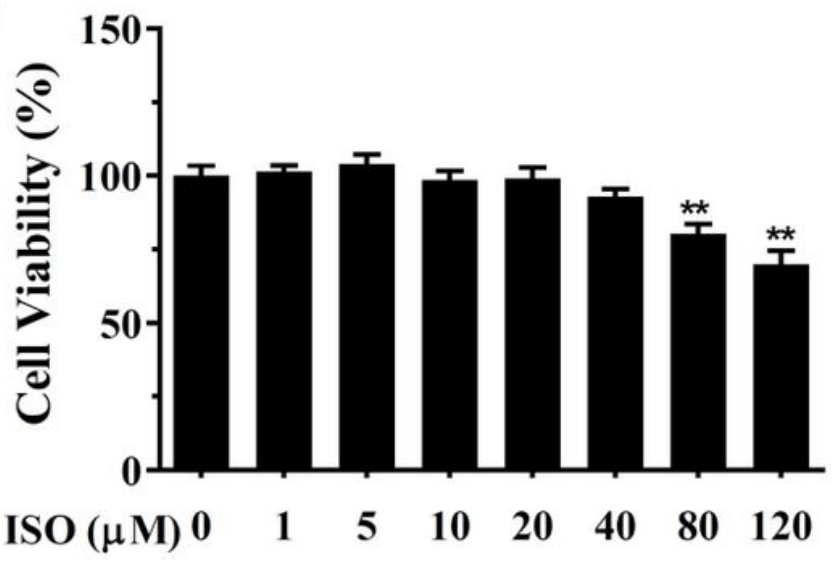

(c)

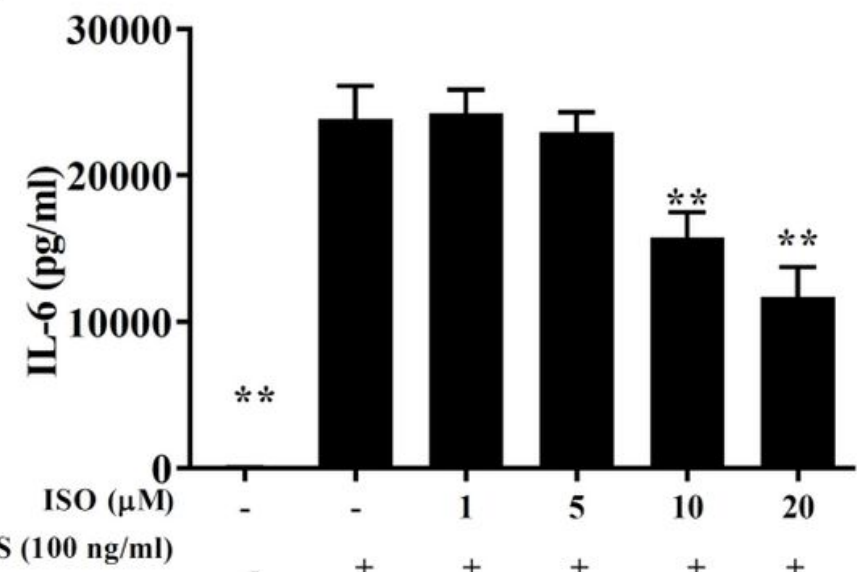

(b)

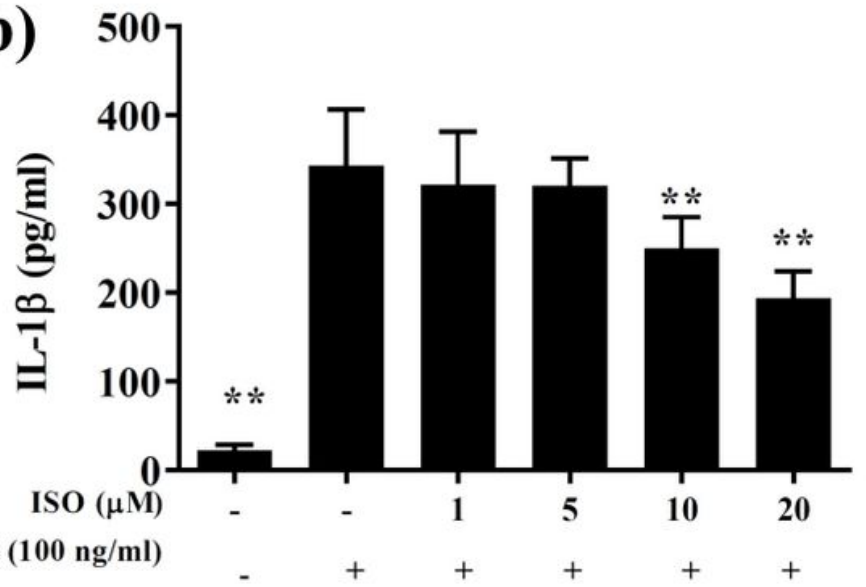

(d)

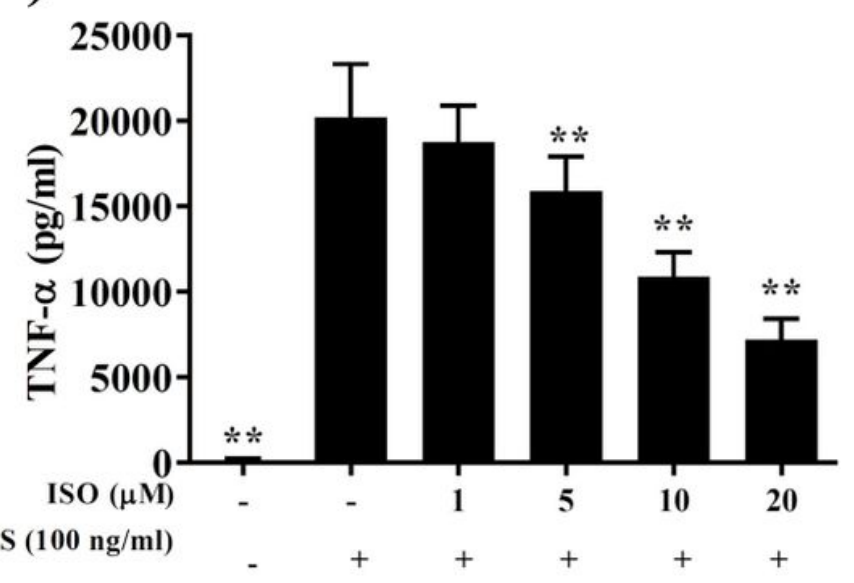

Figure 1

Isorhapontigenin reduced cytokine productions. (a) Cell viability effects of isorhapontigenin (ISO) in RAW 264.7 cells. Isorhapontigenin regulated the (b) IL-1 $\beta$, (c) IL-6, and (d) TNF-a levels in LPS-induced RAW 264.7 cells. Values are mean $\pm S D ;{ }^{*} p<0.05,{ }^{\star *} p<0.01$ vs. LPS-stimulated cells. 
(a)

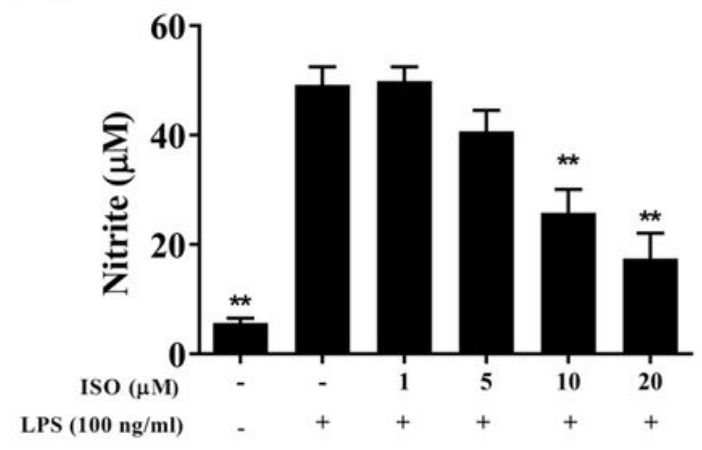

(b)

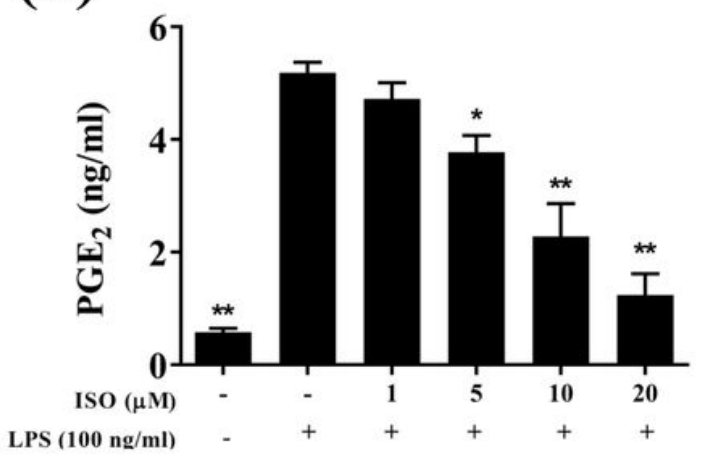

(c)

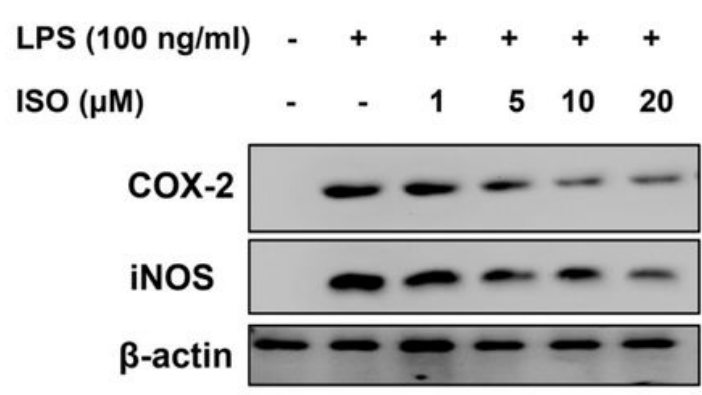

(d)

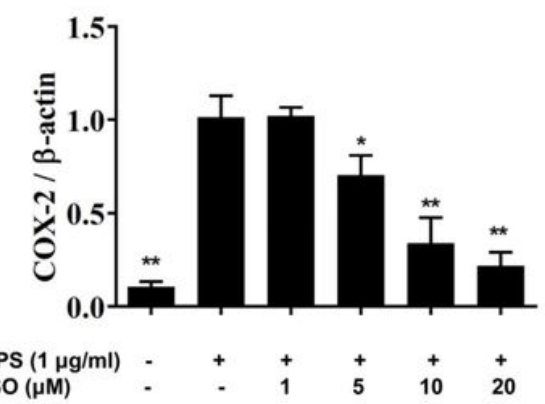

(e)

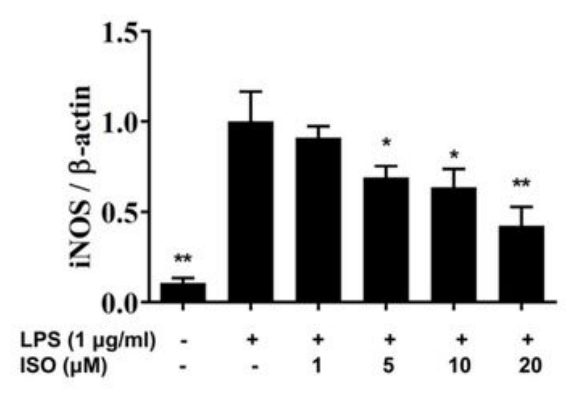

Figure 2

Isorhapontigenin reduced inflammatory mediator productions. Isorhapontigenin (ISO) regulated the (a) nitrite and (b) PGE2 levels in LPS-induced RAW 264.7 cells. (c) ISO regulated iNOS and COX-2 protein expression. The fold-changes in (d) COX-2 and (e) iNOS expressions were measured relative to the $\beta$-actin expression, respectively. Values are mean $\pm S D ; * p<0.05$, ${ }^{\star *} p<0.01$ vs. LPS-stimulated cells.

(a)

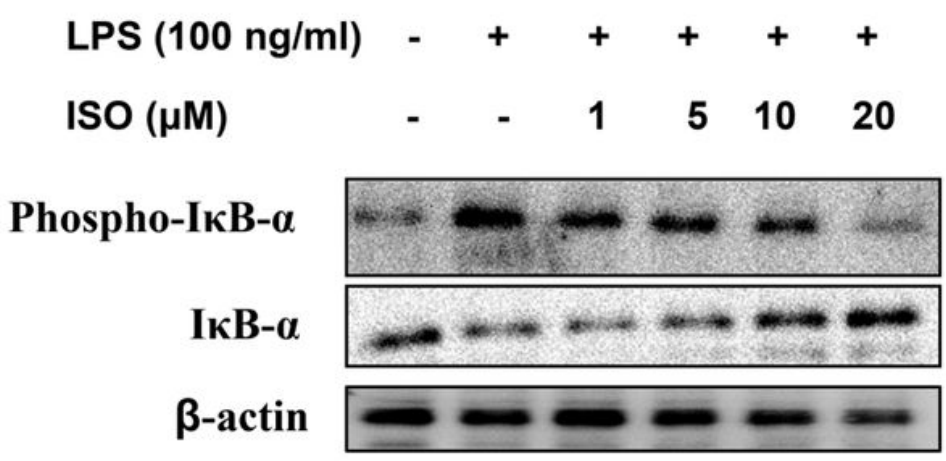

(b)

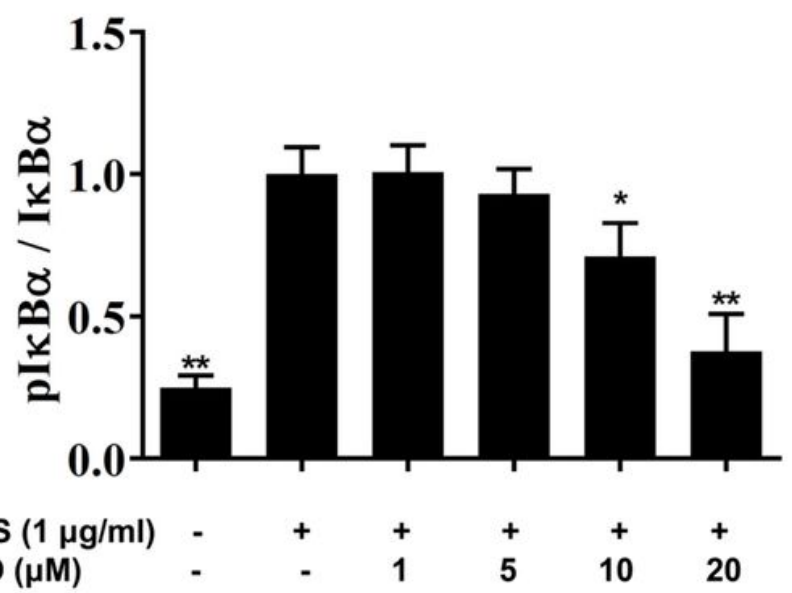

Figure 3 
Isorhapontigenin (ISO) regulated (a)IKB-aphosphorylation, and (b) calculated proportion of phosphorylated IkB-a. Values are mean \pm SD; $p<0.05$, ** $p<0.01$ vs. LPS-stimulated cells.

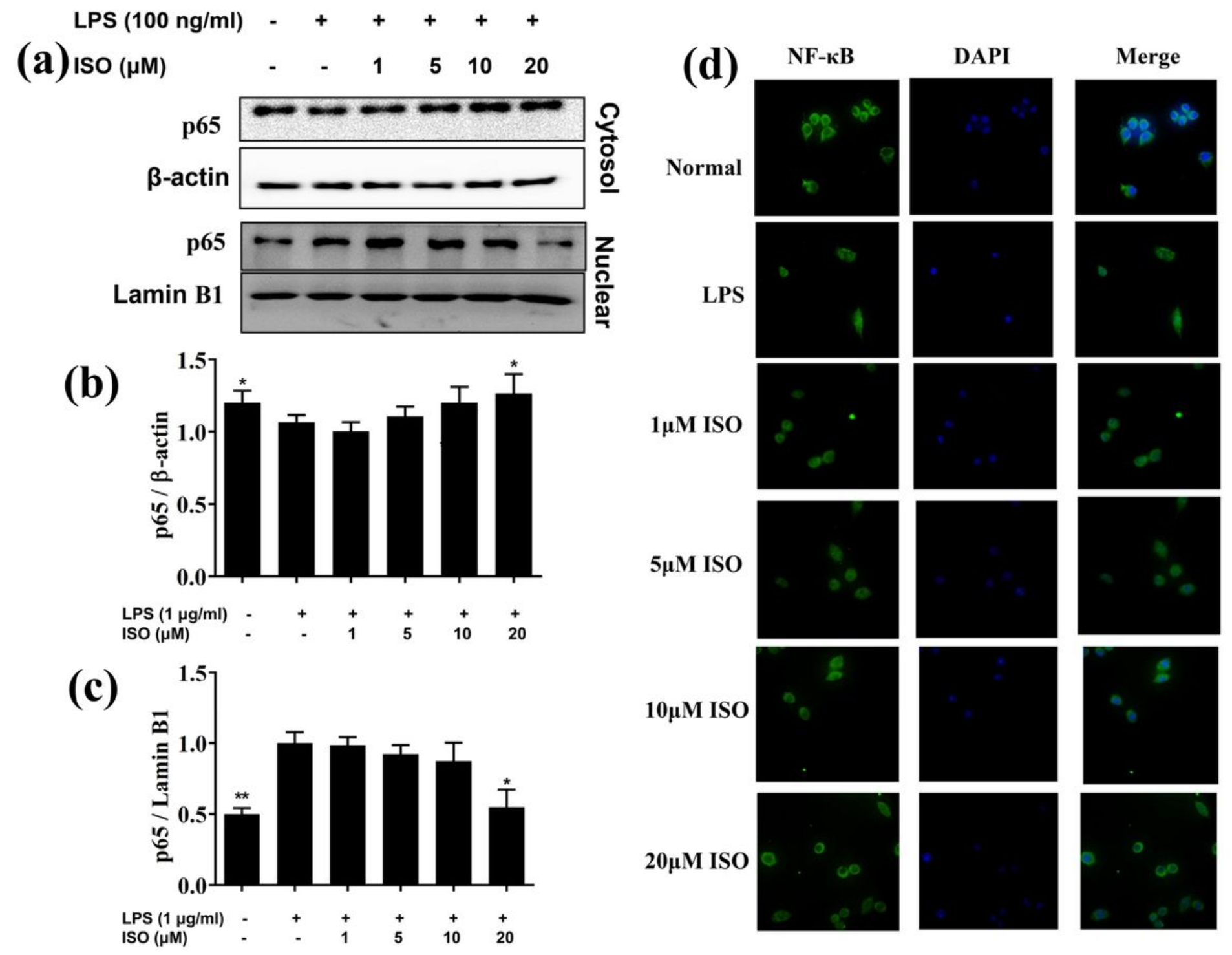

Figure 4

Isorhapontigenin (ISO) regulated nuclear translocation of NF-KB in RAW 264.7 cells. (a) The cytosolic and nuclear levels of p65 were detected by Western blot. (b) The fold changes in cytosolic levels of p65 were measured relative to the $\beta$-actin expression. (c) the fold changes in the nuclear levels of p65 were measured relative to the lamin B1 expression. (d) Fluorescent staining to observe the nuclear translocation of $p 65$. Values are mean $\pm S D ; * p<0.05$, ${ }^{\star *} p<0.01$ vs. LPS-stimulated cells. 
(a)

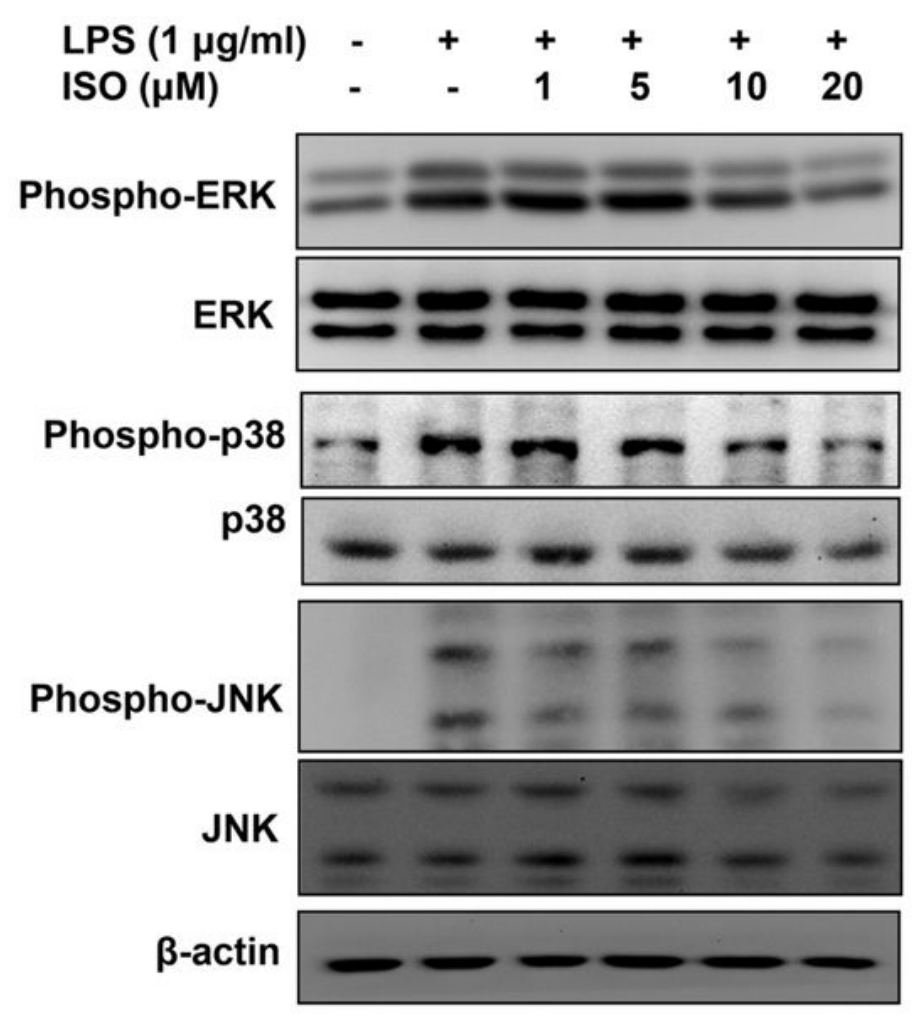

(b)
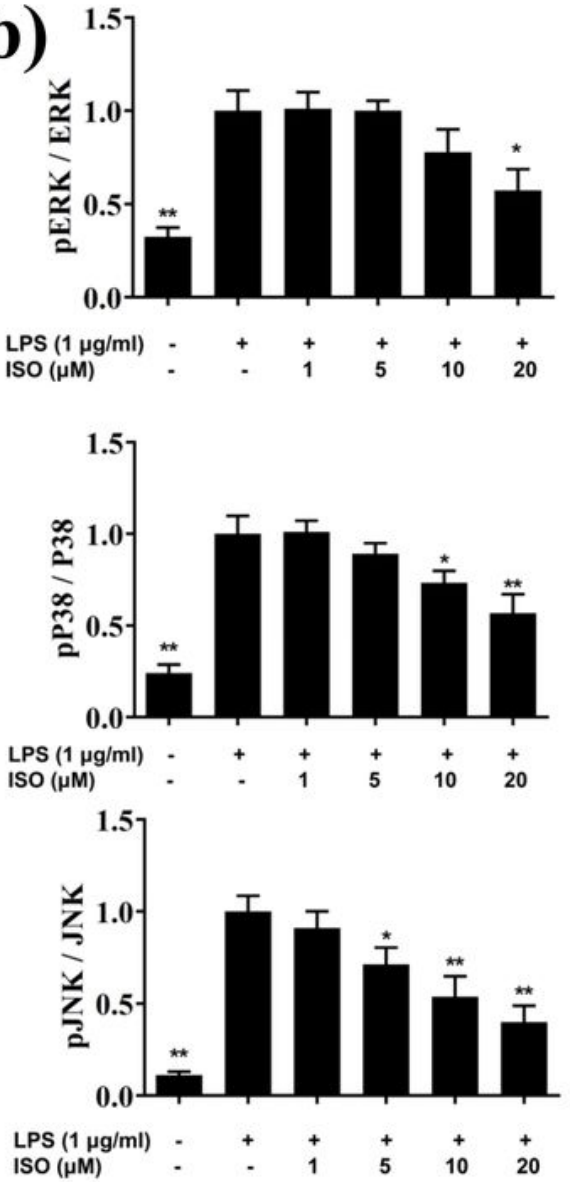

(c)

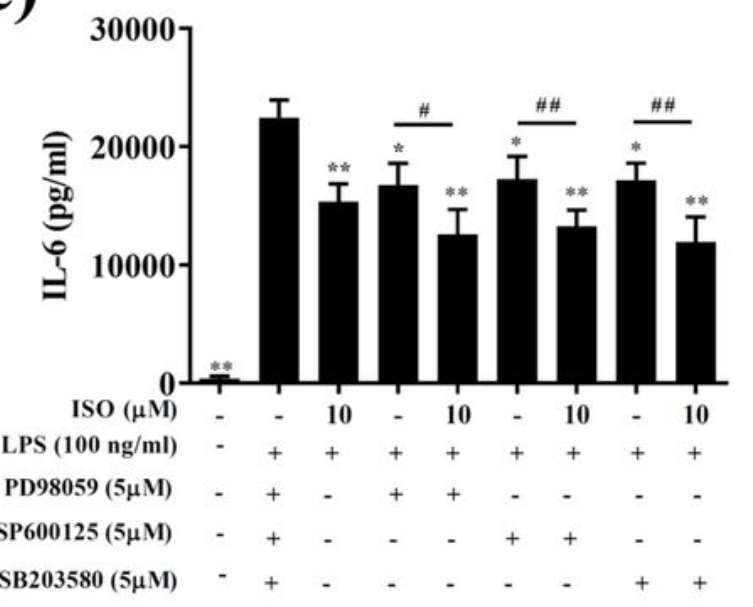

(d)

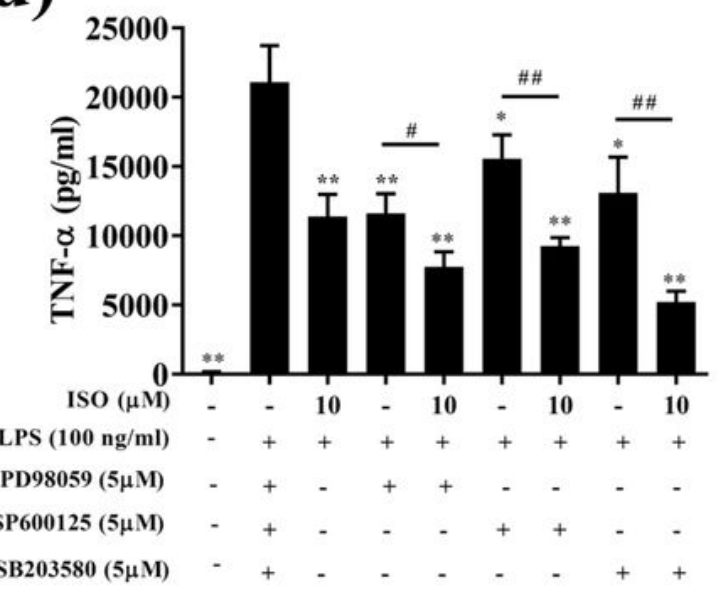

\section{Figure 5}

Isorhapontigenin (ISO) regulated phosphorylation of MAPK. (a) MAPK protein were analyzed by Western blots. (b) The fold changes in protein phosphorylation levels were assayed using densitometry and measured relative to the total levels of each protein. MAPK inhibitors and ISO regulated (c) IL-6, (d) TNF-a levels in RAW 264.7 cells. Values are mean $\pm S D ; * p<0.05$, $* \star p<0.01$ vs. LPS-stimulated cells. \#p<0.05, $\# \# p<0.01$ vs. MAPK inhibitor treated with LPS-stimulated cells. 
(a)
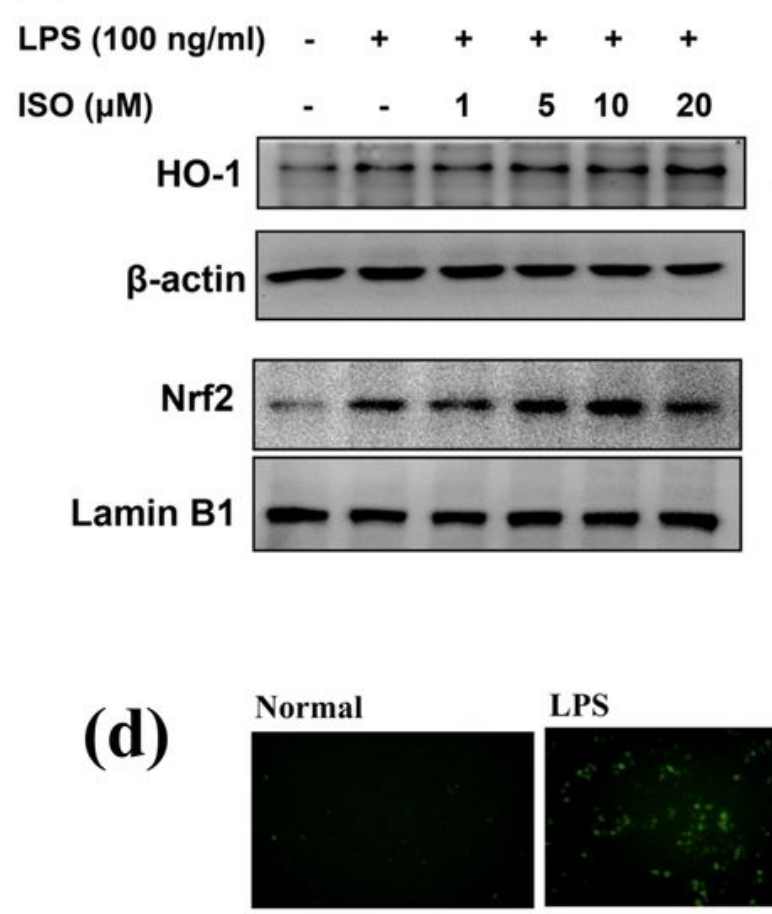

LPS

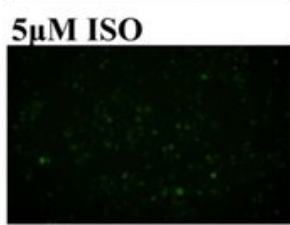

$10 \mu \mathrm{M}$ ISO

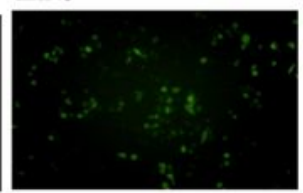

(b)

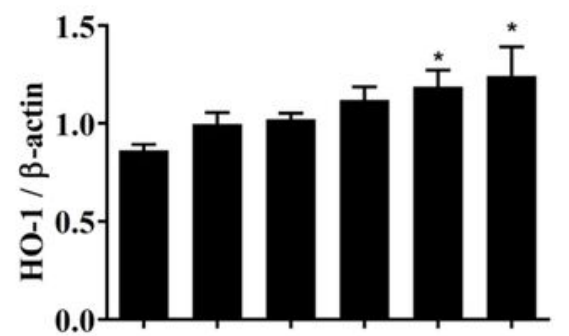

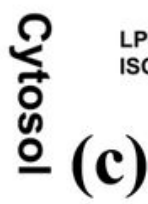
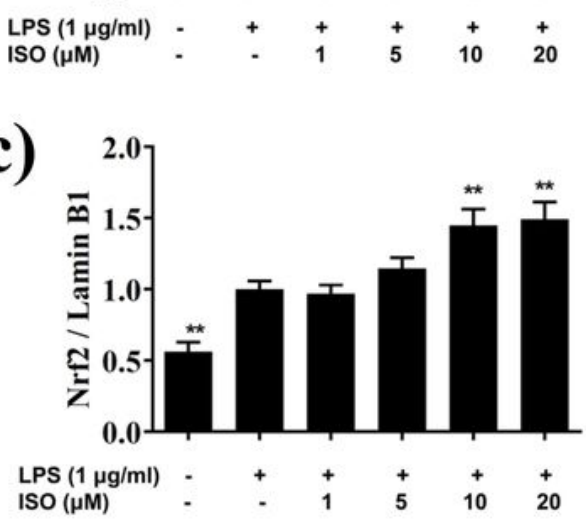

$1 \mu \mathrm{M}$ ISO

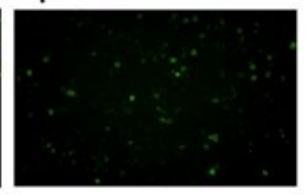

20uM ISO

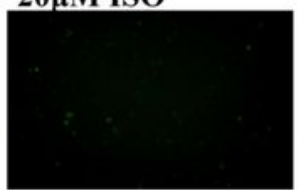

$\mathbf{E}$

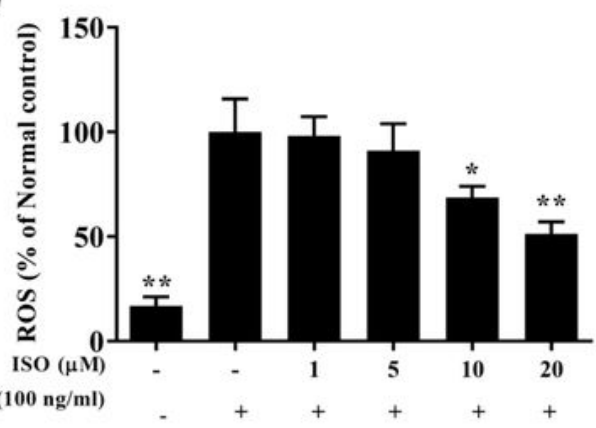

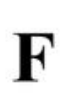

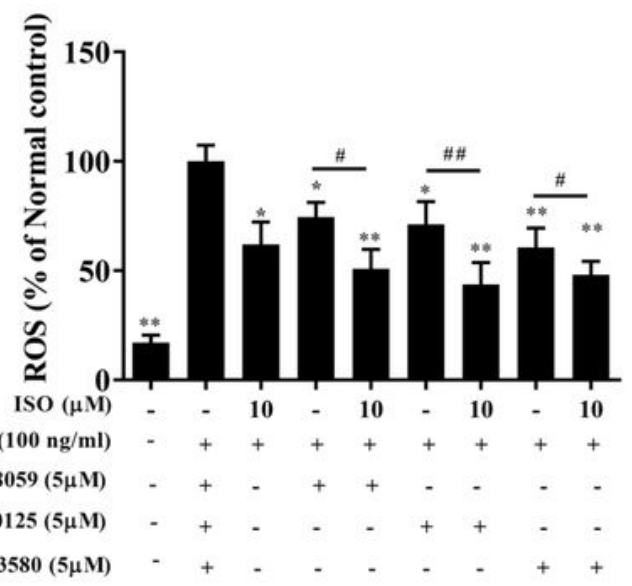

\section{Figure 6}

Isorhapontigenin (ISO) regulated HO-1 and Nrf2 expression in RAW 264.7 cells. (a) The cytosolic levels of HO-1 and nuclear levels of Nrf2 expression were presented by Western blot. (B) The fold changes in cytosolic levels of H0-1 were measured relative to the $\beta$-actin level. (c) The fold changes in the nuclear levels of Nrf2 were measured relative to the lamin B1 level. (d) Isorhapontigenin regulated ROS level, and fluorescence microscopy observed images of intracellular ROS. (e) Percentages of ROS detected in LPS- 
induced RAW 264.7 cells. (f) Inhibitory effects of MAPK inhibitors and ISO on LPS-induced ROS expression in RAW 264.7 cells. The results presented as the mean $\pm S D ; * p<0.05, * \star p<0.01$ vs. LPSstimulated cells. $\# p<0.05, \# \# p<0.01$ vs. MAPK inhibitor treated with LPS-stimulated cells.

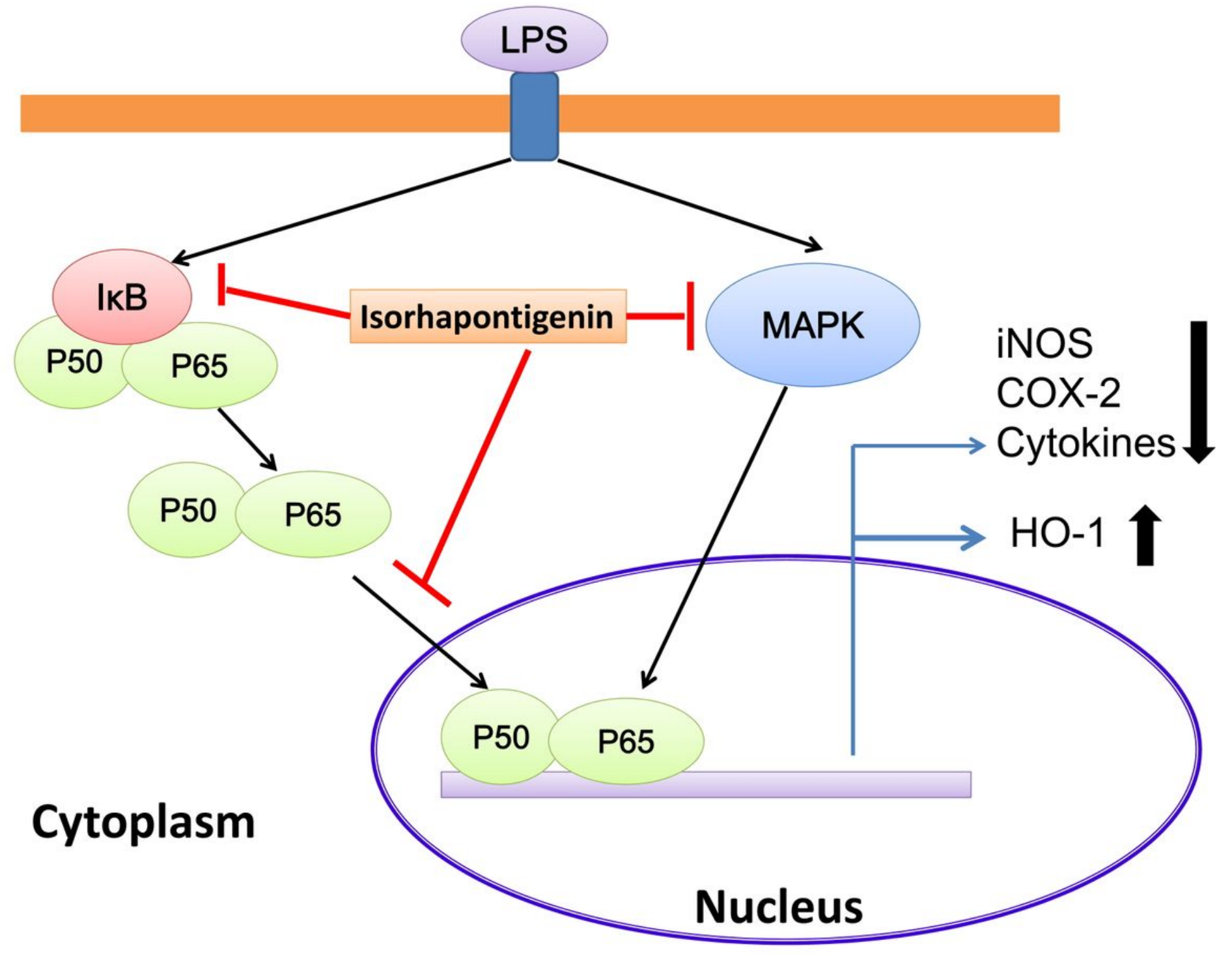

Figure 7

Model explaining the mechanism for the anti-inflammatory effects of isorhapontigenin (ISO) in LPSstimulated RAW 264.7 cells. 